\title{
Measurement-Based LoS/NLoS Channel Modeling for Hot-Spot Urban Scenarios in UMTS Networks
}

\author{
Jiajing Chen, ${ }^{1}$ Xuefeng Yin, ${ }^{1}$ Li Tian, ${ }^{1}$ Nan Zhang, ${ }^{1}$ Yongyu He, ${ }^{1}$ \\ Xiang Cheng, ${ }^{2}$ Weiming Duan, ${ }^{3}$ and Silvia Ruiz Boqué ${ }^{4}$ \\ ${ }^{1}$ College of Electronics and Information Engineering, Tongji University, Shanghai, China \\ ${ }^{2}$ School of Electronics Engineering and Computer Science, Peking University, Beijing, China \\ ${ }^{3}$ Huawei Technology Company, Shanghai, China \\ ${ }^{4}$ Department of Signal Theory and Communications, Polytechnic University of Catalonia, Castelldefels, Spain
}

Correspondence should be addressed to Xuefeng Yin; yinxuefeng@tongji.edu.cn

Received 5 May 2014; Revised 14 July 2014; Accepted 29 July 2014; Published 17 August 2014

Academic Editor: Jose F. Paris

Copyright (C) 2014 Jiajing Chen et al. This is an open access article distributed under the Creative Commons Attribution License, which permits unrestricted use, distribution, and reproduction in any medium, provided the original work is properly cited.

\begin{abstract}
A measurement campaign is introduced for modeling radio channels with either line-of-sight (LoS) or non-line-of-sight (NLoS) connection between user equipment (UE) and NodeB (NB) in an operating universal mobile telecommunications system. A spacealternating generalized expectation-maximization (SAGE) algorithm is applied to estimate the delays and the complex attenuations of multipath components from the obtained channel impulse responses. Based on a novel LoS detection method of multipath parameter estimates, channels are classified into LoS and NLoS categories. Deterministic models which are named "channel maps" and fading statistical models have been constructed for LoS and NLoS, respectively. In addition, statistics of new parameters, such as the distance between the NB and the UE in LoS/NLoS scenarios, the life-distance of LoS channel, the LoS existence probability per location and per NB, the power variation at LoS to NLoS transition and vice versa, and the transition duration, are extracted. These models are applicable for designing and performance evaluation of transmission techniques or systems used by distinguishing the LoS and NLoS channels.
\end{abstract}

\section{Introduction}

Distinctive characteristics of signals coming from a line-ofsight (LoS) or non-line-of-sight (NLoS) path in a wireless environment raised great concerns in mobile radio system design. The probability of whether the LoS path exists in the channel or not depends on various factors, for example, terrain features, building density and locations of users. Statistical models have been employed by the 3GPP spatial channel model (SCM) [1] and the WINNER II SCM-enhanced [2] to describe the LoS existence probability. Recently, characterization of LoS/NLoS channel becomes highly demanded in some applications, such as massive multiple-input-multiple-output (MIMO), mobile back-hauling, user-specific beamforming, and other adaptive techniques used in future heterogeneous networks [3-6]. Newly introduced multipath access scheme-nonorthogonal multiple access (NOMA)for future radio access also relies on the distinction of LoS and NLoS channels [7-9]. Additionally, some ad-hoc wireless communication scenarios and the implementation of some new technologies also prefer the LoS channel, for example, dedicated short range communication (DSRC) system for device-to-device, vehicle-to-vehicle, and machine-tomachine communications [10-15]. Furthermore, the LoS existence has been considered in localization applications, such as the distance estimation between the base station (BS) and the mobile station in [16]. Modeling realistic characteristics of LoS/NLoS channels based on measurements are important for designing and optimizing these techniques or systems.

A problem arising when characterizing the LoS/NLoS channels based on measurements is how to categorize a channel as LoS or NLoS. Detection methods addressed in the literature can be split into two groups, that is, the range-measurement-based and channel-impulse-response- (CIR-) based methods. The former method, such as those introduced in 


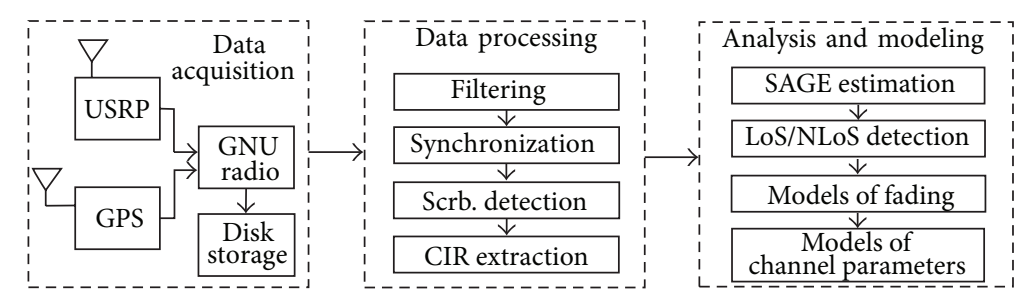

FIGURE 1: A diagram of channel measurement, data analysis, and modeling procedure adopted for LoS/NLoS channel characterization.

[16-18], was adopted for LoS detection in the early stage. The latter approach is mainly based on the shape or statistical properties of the CIRs, for example, the confidence matrix [19], the delay spread [19], the power delay profile (PDP) [20], and the narrowband $K$-factor $[21,22]$. In our contribution, the LoS detection is performed based on the power difference among estimated multipath components, which has been proven to be effective through measurement campaigns.

Some LoS/NLoS propagation models have been reported in the literature. According to measurement environments, these models characterize channels in either indoor or outdoor scenarios. In indoor cases, the distribution of the channel gains for the LoS/NLoS scenarios is presented [23, 24]. Coherence bandwidth and delay spread in LoS/NLoS scenarios have been studied at $5 \mathrm{GHz}[25,26]$ and $60 \mathrm{GHz}$ [27]. In $[28,29]$, statistical models characterizing the shadowing in LoS and NLoS scenarios were extracted from indoor measurements. In outdoor scenarios, path loss models have been attracting more attention [30-34]. Furthermore, statistics of the LoS coverage rate in urban areas for broadband wireless access systems and the LoS existence probability versus elevations of departure of downlink channels have been investigated in $[35,36]$, respectively.

Experiment-based stochastic channel modeling relies on measurement campaigns to be conducted extensively in the environments of interest. Many conventional models have been extracted based on dedicated channel sounding activities. However, due to practical concerns, such as power constraints, poor mobility of the sounding equipment, and limited number of measurement routes, the channel observations do not possess sufficient randomness to characterize the channels ergodically, leading to limited applicability of the resultant models. As an alternative, using in-service public wireless communication systems or networks to measure channels in large areas can be adopted. In [37], a public base station was used as a transmitter, and the received signal strength was measured in the whole coverage of the base station. The advantage of channel measurements in in-service networks is that the channel characteristics observed are identical with those experienced by the user equipment (UE); thus the channel statistics extracted are more realistic. Compared with hundreds of $\mathrm{MHz}$ bandwidth usually adopted in the dedicated wideband channel measurement campaigns, the measurement based on the conventional $2 \mathrm{G}$ public system usually utilizes hundreds of $\mathrm{KHz}$ bandwidth, which facilitates the study of narrowband channel properties, such as the fading-related parameters [38] or performancerelated parameters, for example, the out-of-channel interference [39]. Along with fast developing commercial systems, such as LTE-Advanced, wideband characteristics of channels can be measured based on the in-service networks. Channel modeling based on these data began to attract more attention recently $[36,40]$.

In this work, a receiver was constructed to collect the downlink signals at a hot-spot area of Shanghai in a commercial in-service UMTS network. The impulse responses of the channels are calculated, and a high-resolution estimation algorithm derived based on the SAGE scheme is applied to extract multipath parameters. After appropriately detecting the LoS and NLoS channels by using the novel LoS detection, various aspects of LoS/NLoS channels are investigated and the corresponding models are established based on the extensive observations. They include the deterministic channel maps, which are the geographic map overlaid with spots color-coded by channel characteristics, and statistical models characterizing the channel gain, shadowing, and multipath fading. Furthermore, the statistics of some newly defined channel parameters are studied, which include (i) the distance between NBs and the user equipment in LoS and NLoS conditions, (ii) the life-distance of LoS and NLoS channels, (iii) the LoS existence probability per carrier for a UE, (iv) the LoS existence probability per carrier for a NB, and (v) power variation at LoS-NLoS transition and the transition duration. These models are useful for the design and performance evaluation of communication techniques adaptive to the LoS and NLoS scenarios.

The rest of this paper is organized as follows. Section 2 introduces the measurement equipment, environments, and the specifications applied for data acquisition in an in-service UMTS network. The main steps of processing the measurement data to obtain the estimates of channel impulse responses are also described. In Section 3, the LoS/NLoS detection method is introduced. Section 4 elaborates details of the models established based on the LoS/NLoS channel characteristics obtained. Finally conclusive remarks are addressed in Section 5.

\section{Measurement Equipment, Campaigns, Setup, and Postprocessing}

Figure 1 illustrates the diagram of the data acquisition, processing, and modeling procedure adopted for characterizing LoS/NLoS channels based on the signals of a UMTS network. The equipment used to acquire the signals consists of the following components: a Universal Software-Defined Radio Peripheral (USRP) device of type N210 [41] controlled by the GNU radio software in a computer and a global positioning 


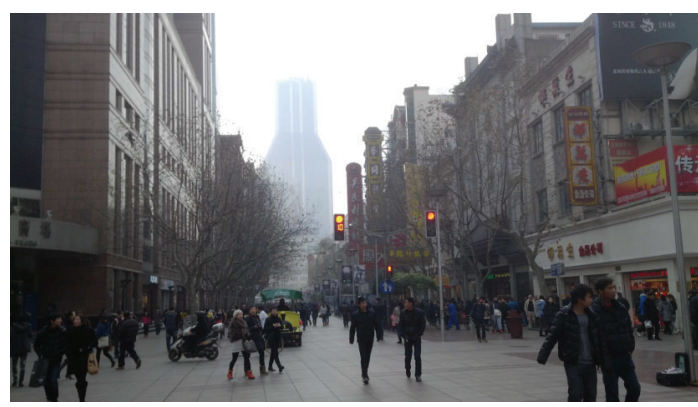

FIgURE 2: A photograph of the Nanjing Road.

system (GPS) module that reports the longitude and latitude of the measurement location to the computer through a serial port, the storage disk, an omnidirectional antenna working in 2-3 GHz UMTS frequency band, and a lead-acid battery. All this equipment was loaded in a trolley moving along predefined routes during the field measurements.

The measurement campaign was performed in the pedestrian zone along Nanjing Road, Shanghai, China. This area is a typical hot-spot of the local UMTS network where complex streets and densely distributed skyscrapers jointly create a city canyon environment. Multiple macro-, micro-, or pico-cellular UMTS stations were deployed in the area. Figure 2 shows a photograph taken on the Nanjing Road during the measurements. Measurements along the streets with different styles of corners provide a large amount of channel observations which are sufficient statistically for modeling the realistic LoS/NLoS channels, including the channel evolution characteristics during the LoS-to-NLoS transitions and vice versa.

During the measurements, the receiver moves at a walking speed of about $1.2 \mathrm{~m} / \mathrm{s}$. Data acquisition was triggered when the receiver departed from its previous location by 5 meters, and the duration of collecting the data at each location is 1 second. The carrier frequency was $2.1376 \mathrm{GHz}$ and the receiving bandwidth was $20 \mathrm{MHz}$. The scrambling code existing in more than 5 consecutive frames is considered to be a valid code. It was found later that the data collected in the whole campaign was from $78 \mathrm{NBs}$, each with a distinctive combination of a scrambling code and a physical carrier.

The raw data collected during the measurements was post-processed in the following five steps.

Step 1 (filtering). The measurement results show that three UMTS carriers were used with central frequencies of $2.1326 \mathrm{GHz}, 2.1376 \mathrm{GHz}$, and $2.1426 \mathrm{GHz}$, respectively. The raw data received with $20 \mathrm{MHz}$ effective bandwidth at the center frequency of $2.1376 \mathrm{GHz}$ is first filtered to obtain three baseband signals $r_{c}(t), c=1,2,3$, for these carriers individually.

Step 2 (synchronization). The beginnings of time-slots in the downlink synchronization channels are detected by correlating the baseband signals with the primary synchronization code (PSC). Multiple NBs are found by checking the dominant peaks in the PDPs of the obtained CIRs. In addition, the estimates of the starting time $t_{0}$ of individual data frame in the common pilot channels (CPICHs) and the index $i$ of estimating scrambling code group are obtained by solving the maximization problem:

$$
\left(\widehat{t}_{0}, \hat{i}\right)=\arg \max _{t_{0}, i}\left|\int_{0}^{T} r_{c}(t)\left(c_{s}^{i}\left(t-t_{0}\right)\right)^{*} d t\right|^{2}, \quad c=1,2,3,
$$

where $c_{s}^{i}(t), i \in[1,2, \ldots, 64]$, consists of 15 secondary synchronization codes permuted according to the $i$ th predefined sequence, $T=10 \mathrm{~ms}$ is the duration of a CPICH frame [42], and $(\cdot)^{*}$ denotes the complex conjugate of given argument.

Step 3 (scrambling code detection). The detection of scrambling code is necessary in our case due to the fact that the information that how the scrambling codes are allocated in the carriers is unknown. The index $j$ of the scrambling code used to modulate the $\mathrm{CPICH}$ is detected by

$$
\widehat{j}=\arg \max _{j \in J_{\hat{i}}}\left|\int_{\hat{t}_{0}}^{\widehat{t}_{0}+T} r_{c}(t) s_{j}^{*}\left(t-\widehat{t}_{0}\right) d t\right|^{2}, \quad c=1,2,3,
$$

where the vector $J_{\hat{i}}=(\hat{i}-1) \cdot 8+[1,2, \ldots, 8]$ contains the indices of 8 scrambling codes in the $\hat{i}$ th group and $s_{j}(t)$ denotes the $j$ th scrambling code. Notice that, for some channels, although the index of scrambling code group can be detected in Step 2, none of the scrambling codes within the group exhibits significantly higher likelihood of being the correct code than the others. This is probably due to the fact that the frequency offset between the NBs and USRP is nonnegligible, resulting in low SNR or signal to interference and noise power ratio (SINR) in the CPICHs. In such cases, the data acquired at this location will not be considered for modeling.

Step 4 (CIR extraction). For the channels identified with the $\hat{j}$ th scrambling code at carrier $c$, the impulse response $h_{c, k}(\tau)$ of the channels observed in the $k$ th $\mathrm{CPICH}$ frame is calculated as

$$
h_{c, k}(\tau)=\int_{\hat{t}_{0}}^{\widehat{t}_{0}+T} r_{c}(t+k T) s_{\hat{j}}^{*}(t-\tau) d t .
$$

Step 5 (parameter estimation). It can be shown that the channel coherence time is beyond the duration of one frame even in the worst case where the people in the environment are moving at the largest speed of $3 \mathrm{~m} / \mathrm{s}$. Thus, the channel observations obtained in one data frame are considered to be coherent and applicable for multipath extraction. A SAGE algorithm is derived and used to estimate the delays and complex attenuations of multiple specular propagation paths from the CIRs observed in individual data frames. Readers may refer to [43] for the derivation of the SAGE algorithm and the steps for implementation. Based on a practical principle that the paths estimated with power $20 \mathrm{~dB}$ lower than the maximum are negligible, we found that 10 paths per CIR are sufficient for reconstructing the dominant portion of the CIRs in most cases. Furthermore, observations not reported 


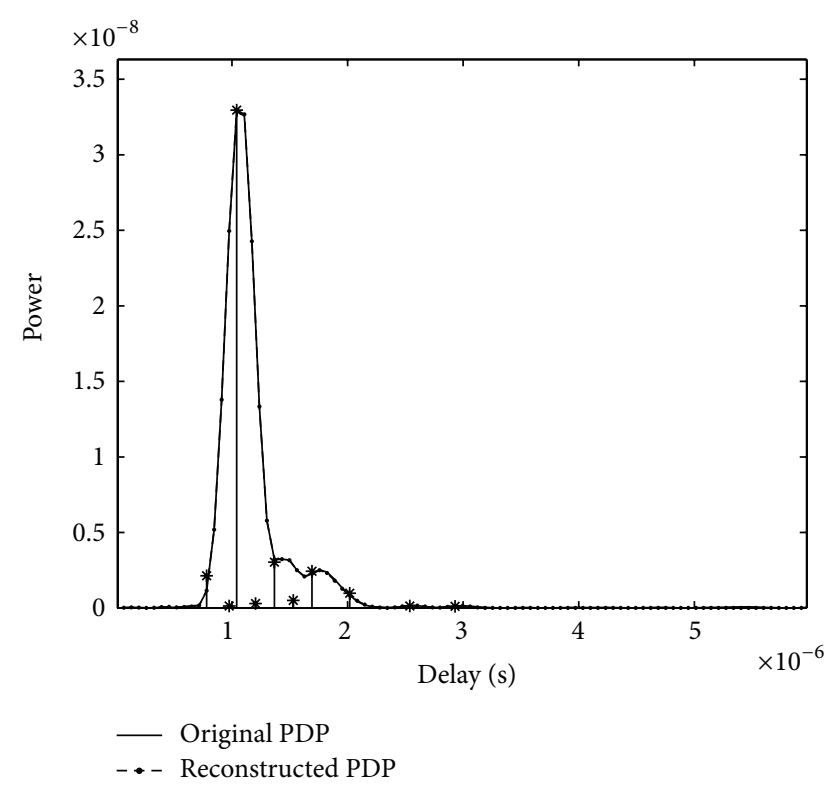

FIgURE 3: An example of an original channel PDP and the PDP of the channel reconstructed based on the multipath parameter estimates. The powers of the estimated paths versus the path delays are also illustrated.

here also show that the parameter estimates usually converge within 30 iterations. Figure 3 depicts the comparison between the original PDP for the channel observed in a CPICH frame and that calculated from the reconstructed signals that are computed based on the estimated 10 specular paths. The stem plot for the powers of the estimated paths with the asteroid marker is also illustrated in Figure 3. It is obvious that the original PDP is well approximated by the reconstructed PDP, indicating that the path components estimated by using the SAGE algorithm can represent the channel impulse response accurately.

\section{LoS/NLoS Detection}

The LoS detection method considered here is based on the estimated multipath parameters. It is a common belief that the LoS path arrives at the UE with the shortest delay. However, due to the limited delay resolution of the communication signals, a LoS path may be combined with NLoS paths which have slightly larger delays than the former. Thus, a multipath cluster can be identified in the vicinity of the true LoS path. This is particularly the case in urban environments where the local clutter around the NB may generate a large amount of diffraction or reflection paths with delays close to the LoS path. Considering these situations, the LoS detection method adopted here is designed for two cases. In the first case where the first-arrival path has the largest power among all estimated paths, a clear decision that the channel is a LoS channel is made without further analysis. In the second case where the first-arrival path does not have the largest power, the deviation $\rho$ between the powers of the strongest path and this first-arrival path is calculated and used to determine the

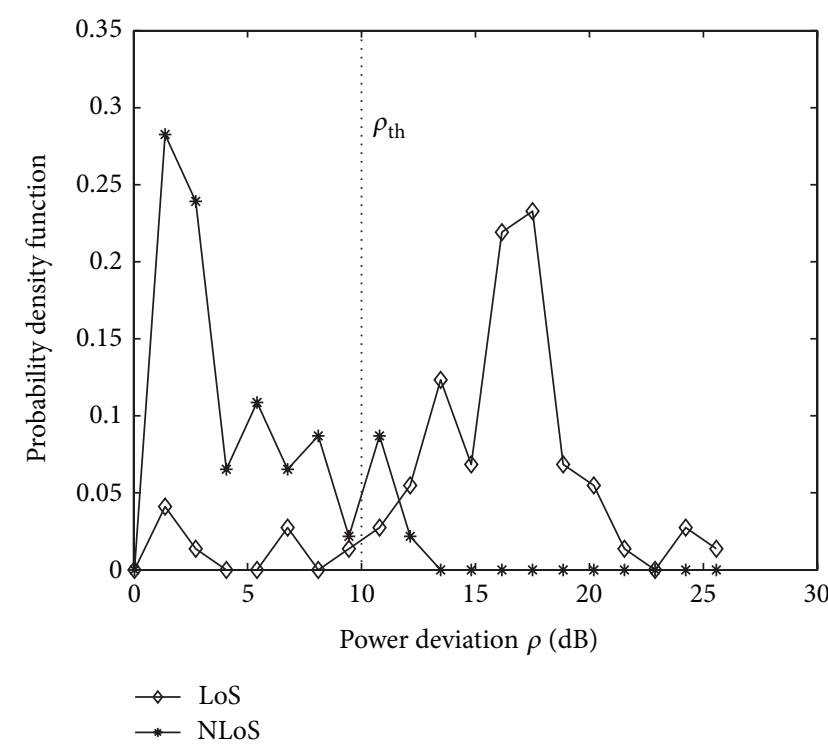

Figure 4: The pdfs of the deviation $\rho$ between the powers of the strongest path and the first-arrival path in the case where the firstarrival path does not have the largest power among all estimated paths.

channel scenarios based on a hypothesis testing. A predefined threshold $\rho_{\text {th }}$ is applied in the testing, where the channel is LoS for $\rho>\rho_{\text {th }}$ and NLoS for $\rho \leq \rho_{\text {th }}$, respectively. The suitable value of $\rho_{\text {th }}$ was identified through the measurements collected at a corner on Nanjing Road, where the receiver moved from LoS to NLoS scenarios. Figure 4 depicts the empirical probability density functions (pdfs) of $\rho$ observed in the true LoS and NLoS cases. It can be observed that the two pdfs interset to overlap around $\rho=9-12 \mathrm{~dB}$. Our calculation shows that, by setting $\rho_{\text {th }}=10 \mathrm{~dB}$, the accumulative probability for detection error in this case can be kept less than 0.1 . Thus, practically, $\rho_{\mathrm{th}}=10 \mathrm{~dB}$ is chosen for the hypothesis testing of a channel being LoS or NLoS in the environment considered here.

It is worth mentioning that the measurement environments were time-variant with people walking around, which can significantly influence the accuracy of LoS detection based on a signal observation. To improve the detection accuracy, it is necessary to select the decision in majority when multiple decisions are available. In the case considered here, the decision with higher occurrence frequency among $49 \mathrm{CPICH}$ frames per location is selected. In most locations, more than 35 frames were observed to provide common LoS detection result.

By using the aforementioned detection method, LoS and NLoS channels observed with valid scrambling codes are identified for all measurement locations. Figures 5(a) and 5(b) demonstrate an example of the LoS and NLoS channel maps for Nanjing Road where the geometric map or the photograph of the environment is overlaid with spots marking the channel categories observed by the UE. Notice that since there are many NBs deployed along the Nanjing Road transmitting signals with different scrambling codes at three carriers, Figure 5 illustrates the LoS detection results at all measured 


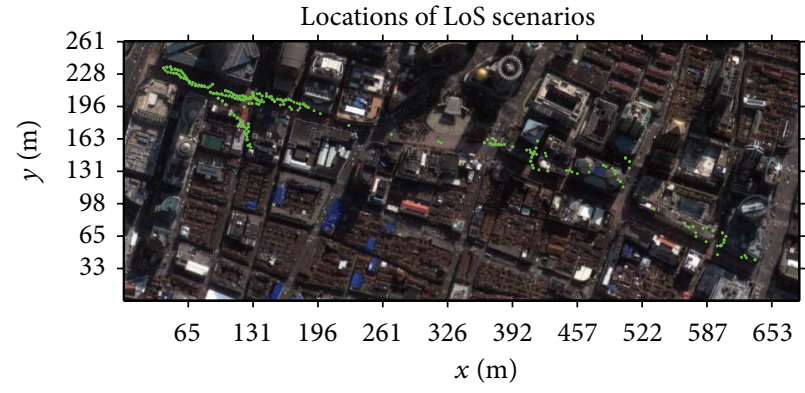

(a) Locations where LoS channels are observed

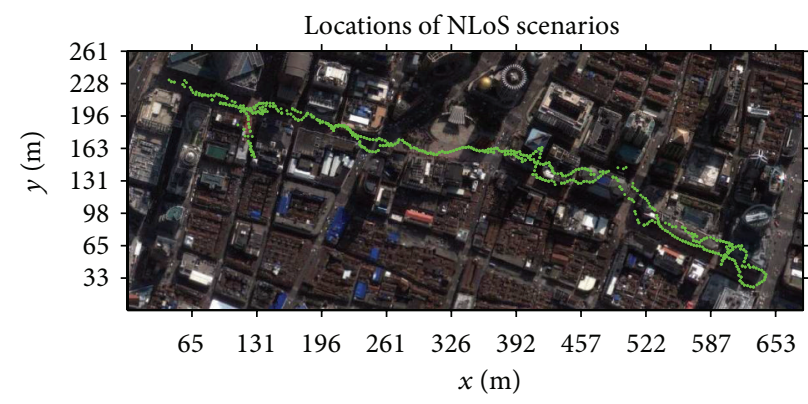

(b) Locations where NLoS channels are observed

FIgURE 5: The locations where the channels are found to be LoS and NLoS channels along the Nanjing Road.

locations for the channel with the largest power among all valid channels identified. It can be observed that more LoS channels are found than the NLoS channels at the left end of the Nanjing Road. This is reasonable as that area is an open cross with two main roads intersect. Furthermore, NLoS channels are widely observed along the Nanjing Road, a typical street canyon environment where the diffraction and the reflections from skyscrapers are the major propagation mechanisms.

\section{LoS/NLoS Channel Models}

In this section, various aspects of the LoS and NLoS channels are analyzed, and the corresponding stochastic models are established based on 664 LoS and 1181 NLoS channel observations. These characteristics include the conventional fading statistics and the distributions of some novel parameters. For notational convenience, we use $h_{\mathrm{LoS}, m}(\tau)$ and $h_{\mathrm{NLoS}, n}(\tau)$ to denote the CIR of the $m$ th observation of LoS channel and the $n$th observation of NLoS channel, respectively. The corresponding narrowband channel coefficients $h_{\mathrm{LoS}, m}$ and $h_{\mathrm{NLoS}, n}$ are calculated as $h_{\mathrm{LoS}, m}=\int h_{\mathrm{LoS}, m}(\tau) d \tau$ and $h_{\mathrm{NLoS}, n}=$ $\int h_{\mathrm{NLoS}, n}(\tau) d \tau$, respectively. The channel gains $P_{\mathrm{LoS}, m}$ and $P_{\mathrm{NLoS}, n}$ represented in $\mathrm{dB}$ are calculated as $P_{\mathrm{LoS}, m}=$ $20 \log _{10}\left|h_{\mathrm{LoS}, m}\right|$ and $P_{\mathrm{NLoS}, n}=20 \log _{10}\left|h_{\mathrm{NLoS}, n}\right|$, respectively.

4.1. Channel Gain, Shadowing, and Multipath Fading. The channel gain $P_{\mathrm{LoS}, m}$ can be written as $P_{r}=P_{t}-P_{p}+P_{s}+P_{m}$ where $P_{t}, P_{p}, P_{s}$, and $P_{m}$ represent the transmission power, path loss, shadowing, and multipath fading denoted in $\mathrm{dB}$, respectively. Figure 6 depicts the empirical cumulative distribution functions (cdfs) of the channel gains $P_{\mathrm{LoS}, m}$ and $P_{\mathrm{NLoS}, n}$, respectively. It can be observed that the channel gains for LoS are concentrated in the region more to the right of the abscissa than to the NLoS scenario. Different kinds of distributions including the $m$-Nakagami, Rice, Weibull, normal, and $\alpha-\mu$ are used to fit with the empirical results. The Kolmogorov-Smirnov test (K-S test) has been conducted for evaluating the consistency of the analytical pdf and the empirical graphs. The results show that both cdfs are well fitted by normal distributions with appropriately chosen parameters reported in Table 1. It can be observed from the table that the average of $P_{\mathrm{LoS}, m}$ is $6.12 \mathrm{~dB}$ higher than that in the NLoS

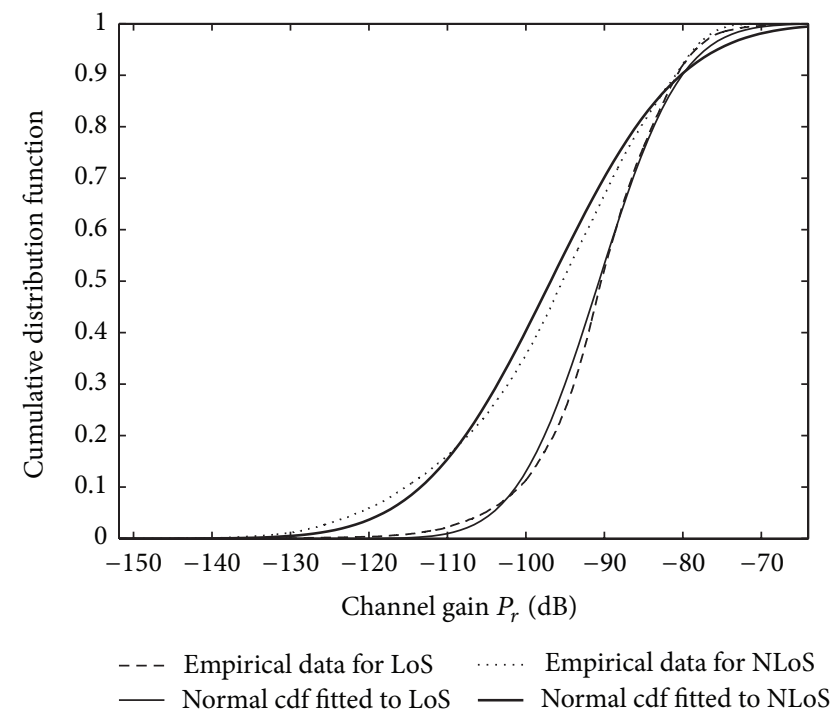

Figure 6: The empirical and fitted cdfs of the channel gains in the LoS and NLoS scenarios.

TABle 1: Parameters of analytical pdfs fitted to the empirical data.

\begin{tabular}{lcc}
\hline & \multicolumn{1}{c}{ LoS scenarios } & NLoS scenarios \\
\hline $\begin{array}{l}\text { Channel gain } \\
\text { (normal) }\end{array}$ & $\mu=-90.68, \sigma=8.27$ & $\mu=-96.80, \sigma=12.92$ \\
Shadowing $(\alpha-\mu)$ & $\alpha=0.9184, \mu=4.5918$ & $\alpha=1.6327, \mu=0.9184$ \\
Multipath $(\alpha-\mu)$ & $\alpha=2.4490, \mu=2.1429$ & $\alpha=2.5510, \mu=1.0204$ \\
\hline
\end{tabular}

scenario, and the standard deviation of the channel gain in the LoS scenario is $4.65 \mathrm{~dB}$ less than that in the NLoS scenario. These statistical differences indicate that the existence of the LoS path in the channel can not only bring $6 \mathrm{~dB}$ gain in average for the received signal but also reduce the variation of the channel gain significantly.

To calculate the gain due to the shadowing $P_{s}$ which attributes to the large structures around the receiver, it is necessary to subtract the path loss $P_{p}$ caused by free-space propagation and the terrain features. In our case, since the exact locations of the NBs are unknown, the path loss may not be calculated analytically by using existing models. As 


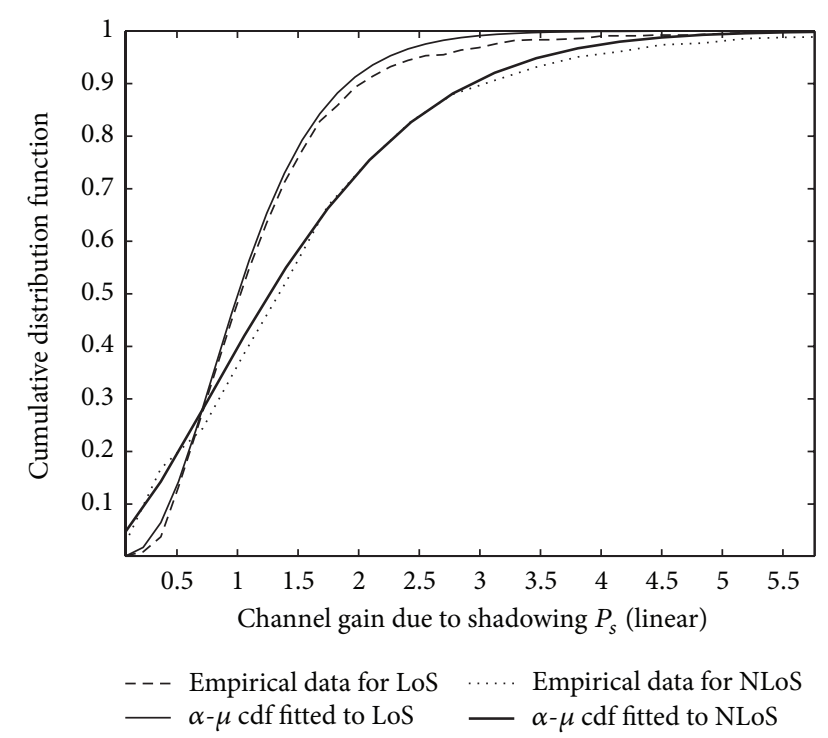

FIGURE 7: The empirical and fitted cdfs of shadowing in the LoS and NLoS scenarios.

an alternative, the empirical path loss is computed in the following manner. For any location, say $A$, channel gains observed in the surrounding locations within 50-m radius are averaged to obtain the estimate of the path losses $P_{p, \text { LoS }}$ and $P_{p, \mathrm{NLOS}}$ at $A$ for LoS and NLoS scenarios, respectively. The shadowing of a LoS channel $P_{s, \text { LoS }}$ at the location $A$ is then calculated by taking the mean of $P_{\mathrm{LoS}, m}-P_{p, \mathrm{LoS}}$, where $P_{\mathrm{LoS}, m}$ is the channel gain observed in a LoS scenario at any location less than $10-\mathrm{m}$ deviation from $A$. Similar calculations are applied to calculate the shadowing of a NLoS channel $P_{s, \mathrm{NLoS}}$.

Figure 7 depicts the empirical cdfs of the shadowing in the LoS and NLoS scenarios, respectively. The cdfs of $\alpha$ $\mu$ distributions which were found to fit the best with the empirical graphs among multiple candidates are also illustrated in Figure 7. The parameters of the pdfs of the adopted distributions are reported in Table 1. It can be observed from Figure 7 that the mean of the gain due to shadowing both in LoS and NLoS scenarios are around 1 in linear scale, which indicates that the path loss has been correctly removed in the calculation. The spread of the shadowing is larger in the NLoS channels than that in the LoS channels. This observation shows that more complex environments exist around the receiver in the NLoS scenarios, which create larger variation of the shadowing than in the LoS scenarios.

Multipath fading in a channel can be calculated by subtracting the path loss and shadowing from the over channel gain. Figure 8 depicts the empirical cdfs of channel gain attributed to the multipath fading in the LoS and NLoS scenarios, respectively. The $\alpha-\mu$ distributions which were shown by the K-S test to fit the best to the empirical graphs are also depicted in Figure 8. It can be observed that the multipath fading magnitudes in the linear scaling are distributed within the interval of $[0,3]$ with mean values equal to 1 for both scenarios. Furthermore, the cdf is more concentrated in the LoS than in the NLoS scenarios, indicating that the variation of the multipath fading is more severe in NLoS scenarios. Table 1

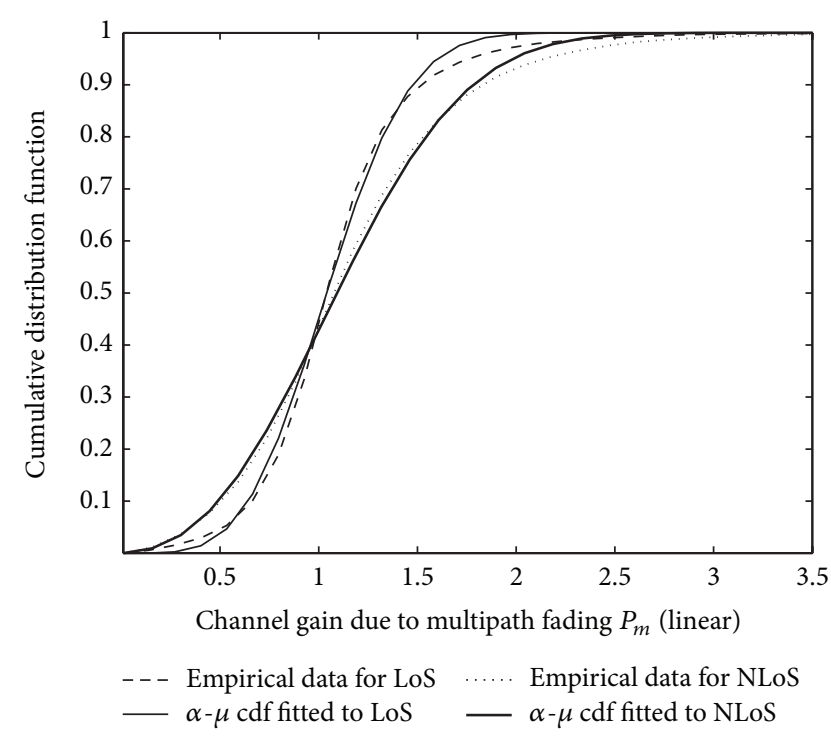

Figure 8: The empirical and fitted cdfs of multipath fading in the LoS and NLoS scenarios.

reports the parameters of the $\alpha-\mu$ cdfs that fit well with the empirical data.

4.2. Distance between the NB and the UE. As the locations of the NBs are unknown in our case, it is difficult to establish the path loss models for both LoS and NLoS scenarios. However, the distributions of the path loss, which can be calculated based on a method that will be introduced later, are applicable to deducing the statistics of the distances between NBs and UE in either LoS or NLoS cases. Such information is considered to be useful for channel simulations and designing the transmission power for NBs and UE with respect to the LoS and NLoS scenarios.

Here $P_{t}$ can be considered to be deterministic, $P_{p}$ depends on the direct distance $d$ between the NB and the UE, and $P_{s}$, $P_{m}$ are uncorrelated random variables. In the case considered here, the unknown $d$ can be viewed as a random variable, and consequently $P_{p}$, as a deterministic function of $d$, is a random variable as well. The pdf of $P_{r}$ is the convolution of the pdfs of $-P_{p}, P_{s}$, and $P_{m}$. Under the condition that the empirical pdfs of $P_{s}$ and $P_{m}$ are known, we can calculate the pdf of $P_{p}$ by the deconvolution operation. Figure 9 depicts the estimated empirical pdf of the $P_{p}$ in LoS and NLoS scenarios. It is worth mentioning that, since $P_{t}$ is unknown, the absolute value of the abscissa of Figure 9 may be incorrect. It can be observed that the mean path loss is $7 \mathrm{~dB}$ less in LoS than in NLoS scenarios and the spread of the path loss is larger in NLoS than in LoS scenarios.

Since the path loss is a deterministic function of the distance $d$ between the NB and the UE, we may deduce the distribution of $d$ from the estimated pdf of $P_{p}$. For simplicity, the following path loss model [44, Section 2.6] is used:

$$
P_{p}=-10 \gamma \log _{10}\left(\frac{d}{d_{0}}\right)+20 \log _{10}\left(\frac{\lambda}{4 \pi d_{0}}\right)
$$




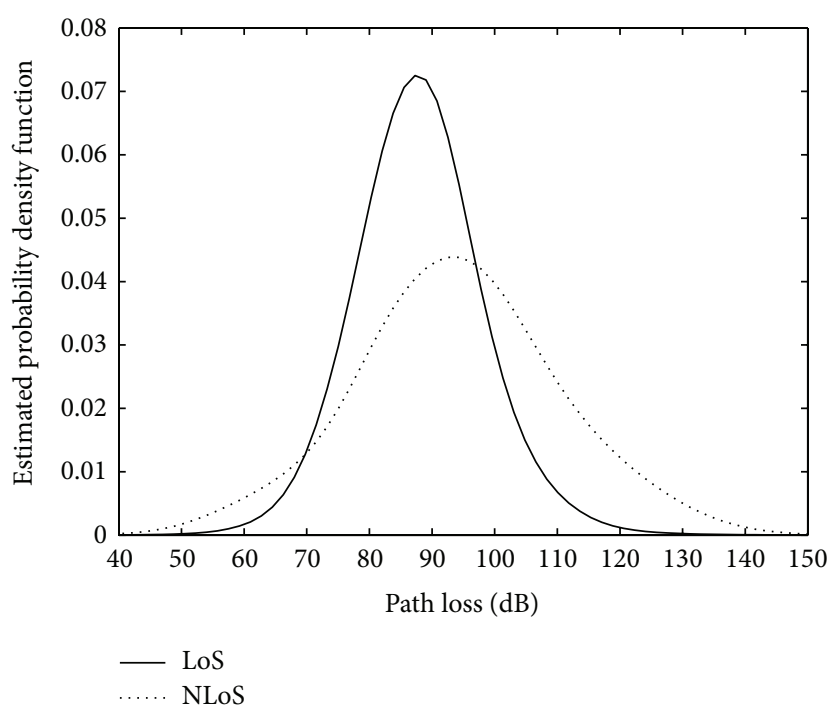

FIGURE 9: The empirical pdfs of estimated path loss in LoS and NLoS scenarios.

where the path loss exponent $\gamma$ should be selected within $[2.7,3.5]$ and the reference distance $d_{0}$ is between 10 and $100 \mathrm{~m}$ for urban micro-cellular environments. In our case, $\gamma=3$ and $d_{0}=50$ are selected, which can be changed if more accurate path loss model is available for the environment considered. By assuming that the transmit power $P_{t}=0 \mathrm{~dB}$, the pdf of $d$ is obtained from the empirical pdf of $P_{p}$ by replacing $P_{p}$ with (4). Figure 10 illustrates the resultant pdfs of direct distance from the NB to UE in LoS and NLoS scenarios, respectively. It can be observed from Figure 10 that the LoS users are maximum $2.5 \mathrm{~km}$ from the NB. The most of the LoS users are located at $200 \mathrm{~m}$ from the NB. The NLoS users are widely spread within the range from 0 to $4 \mathrm{~km}$, and their majority is around $300 \mathrm{~m}$ from the NBs. The nonzero probability observed for large range up to $4 \mathrm{~km}$ indicates that this may be due to the existence of macro-cellular NBs. These results clearly demonstrate that the LoS users are located closer to the NB than the NLoS users. In other words, the NBs with small coverage areas tend to serve more LoS users than the NBs with larger coverage ranges in the Nanjing Road environments.

4.3. Life-Distance of LoS Channel. To implement, for example, the user-specific beamforming technique, a UE is usually expected to experience LoS scenarios in a long distance. Thus, it is important to investigate the statistics of the so-called "lifedistance of the LoS/NLoS channel" $d_{c}$ within which a UE experiences the LoS channel constantly. Based on the location information of the measured spots, the measurement routes are accurately reproduced. In the case where the LoS or NLoS channel appears in a certain number of consecutive locations, $d_{c}$ can be calculated as the displacement from the first to the last location. Figure 11 illustrates an example of the fragments where LoS or NLoS channels were constantly observed for the NB with the scrambling code number 188 . In this example, the maximum of the life-distance of LoS channel is calculated as

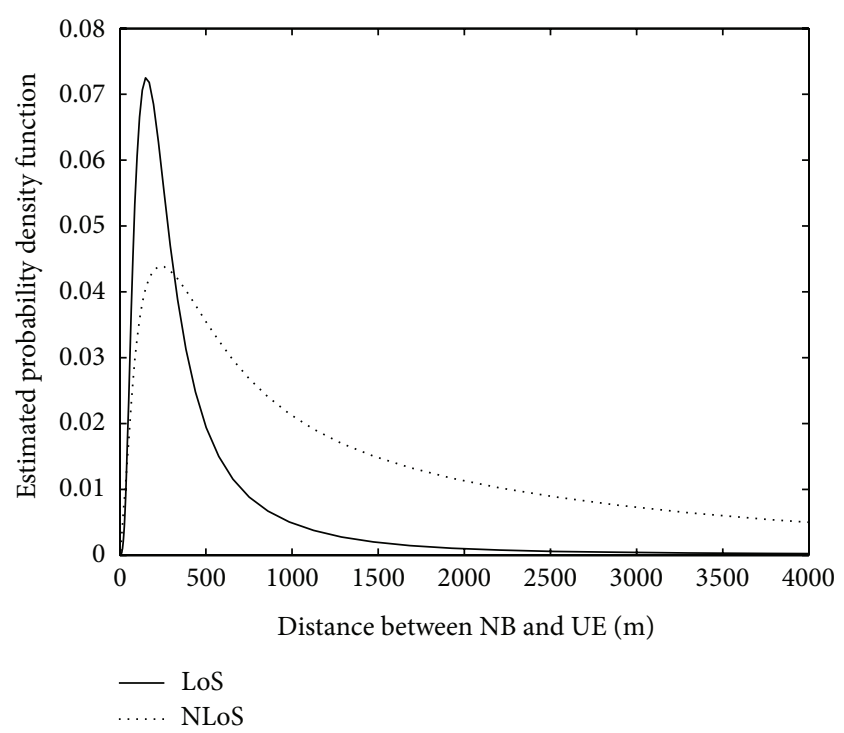

FIGURE 10: The empirical pdfs of the distance between the NB and the UE in LoS and NLoS scenarios.

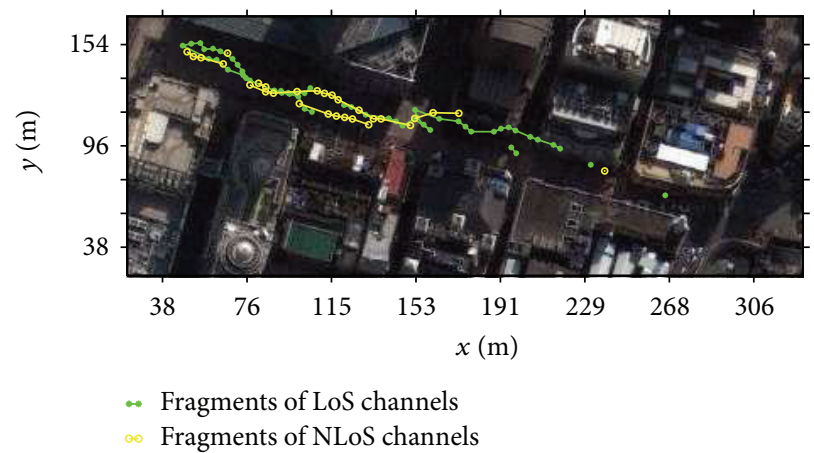

FIGURE 11: An example of calculating life-distance of LoS and NLoS channels for a NB with the scrambling code number 188.

25 meters which was found along the spacious street. Notice that, due to the limited GPS resolution and the fixed distance of $5 \mathrm{~m}$ between adjacent measured spots, the minimum lifedistance of LoS or NLoS channel is lower-bounded by $5 \mathrm{~m}$.

Figure 12 depicts the cdf of the life-distance of LoS and NLoS channels calculated based on the observations of totally 148 fragments for LoS and 298 fragments for the NLoS channels. It can be observed from Figure 12 that the longest LoS and NLoS life-distance can be up to $28.1 \mathrm{~m}$ and $160 \mathrm{~m}$, respectively. Furthermore, nearly $50 \%$ of the LoS and NLoS life-distances observed are 5 meters, and $20 \%$ of the LoS and NLoS life-distances are within the range of 5 to 10 meters. The majority of the life-distances observed are below 10 meters which indicates that the LoS and NLoS scenarios transit frequently when a UE walks on the city canyon environments as in the case of Nanjing Road.

4.4. LoS Existence Probability for a UE and for a NB. The probability of a UE experiencing the LoS channel at an arbitrary location in the network is of interest to know. Considering 


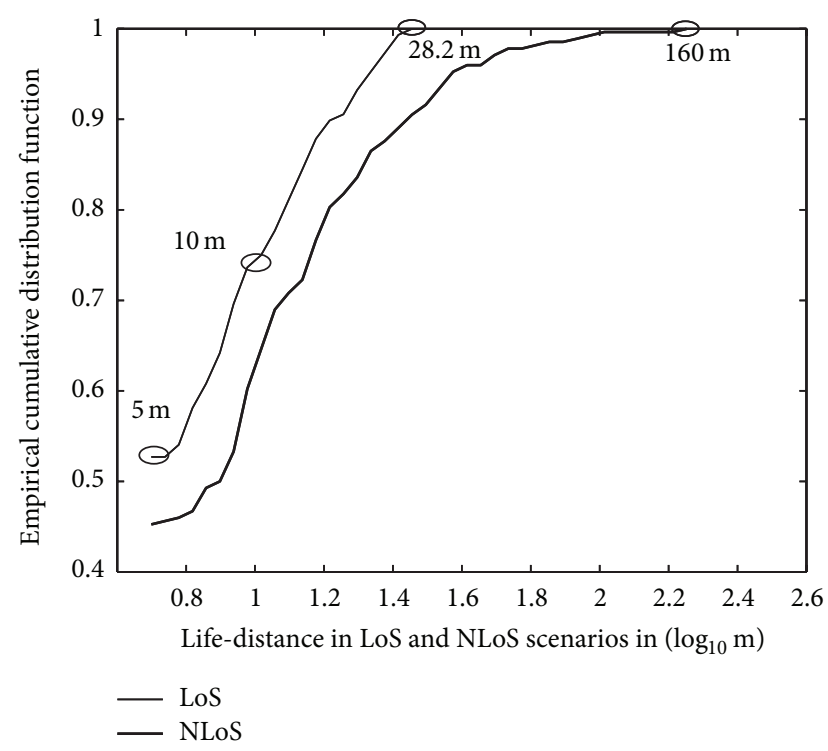

FIGURE 12: The empirical cdfs of the LoS and NLoS life-distance.

that the UE may receive signals from more than one NB, the "LoS existence probability for a UE" $P_{\mathrm{LoS} / \mathrm{UE}}$ is defined as the percentage of the NBs with the fact that the UE can set up a LoS channel among all valid NBs. In the measurement data, the case of receiving two scrambling codes per carrier is the majority. The $P_{\mathrm{LoS} / \mathrm{UE}}$ for the two-scrambling-code cases is investigated based on available observations. Figure 13 depicts the empirical percentage of $P_{\mathrm{LoS} / \mathrm{UE}}$ for three carriers. It can be observed that the distributions of $P_{\mathrm{LoS} / \mathrm{UE}}$ are similar for all three carriers, which is probability due to the fact that every NB in Nanjing Road makes use of three carriers. Furthermore, the case of no LoS path existed to the two NBs has the probability larger than 0.4 . The opposite situation that all NBs have LoS connections to UE has the probability around 0.15 . It is easy to deduce that the case where the UE has at least one LoS connection to the surrounding NBs can happen with probability 0.6 . These results may be used to provide probabilistic assumptions for designing the cooperative multipoint (CoMP) techniques when the coexisting multilinks involve both LoS and NLoS channels.

For individual NBs, it is important to know the distribution of a specific percentage of UE that are categorized as LoS users among all UE served by the NB. The "LoS existence probability per NB" $P_{\mathrm{LoS} / \mathrm{NB}}$ is defined as the ratio of number of LoS UE to the number of all UE in the coverage of a NB. Figure 14 illustrates the empirical percentages of LoS existence probability per NB per carrier, calculated based on the observations in total 78 NBs. It can be observed from Figure 14 that, for three carriers, the situation of all UE in a $\mathrm{NB}$ experiencing LoS channel is zero, the situation in which $20 \%$ of the UE in a NB are LoS users occurs with 0.4 probability, and the case with $80 \%$ of the UE being LoS users happens with probability close to 0.2 . This result indicates that, on the Nanjing Road, the existing NBs can be categorized into two groups, one with the most users being LoS users and the other with the most users being NLoS

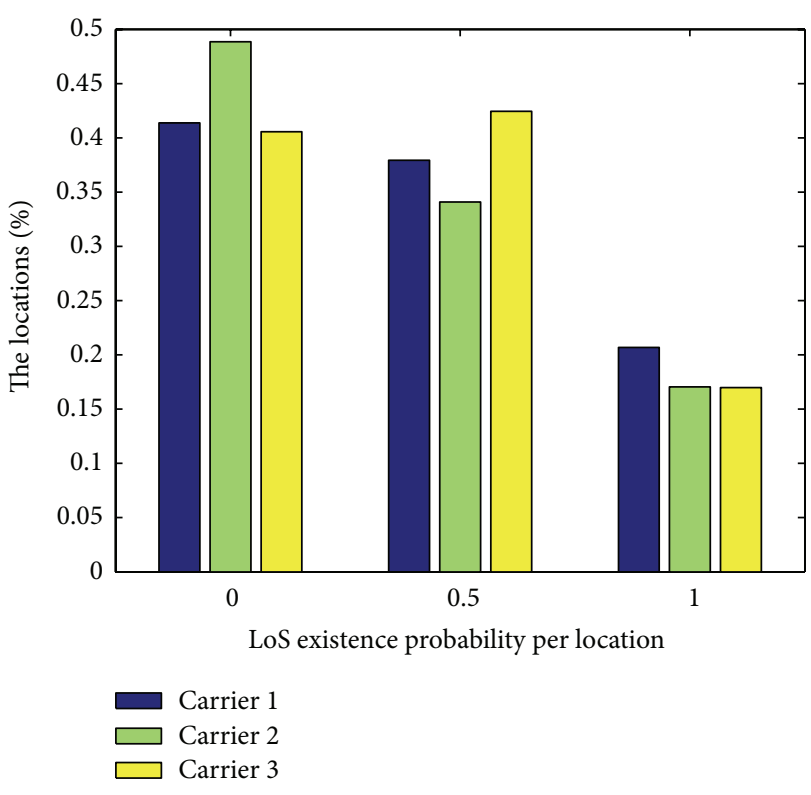

FIGURE 13: The empirical percentage of LoS existence probability per location.

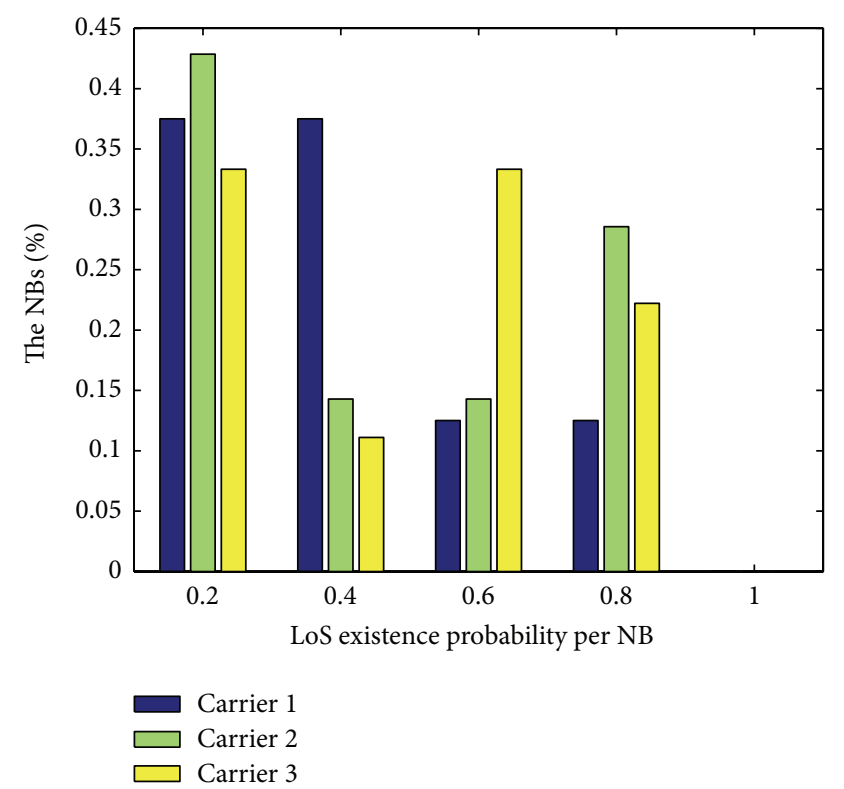

FIGURE 14: The empirical percentage of LoS existence probability per NB.

users. This categorizing scheme probability is due to the fact that the most NBs deployed on Nanjing Road are either micro- or pico-cellular NBs. Depending on the type of the $\mathrm{NB}$, distinctive distribution of "LoS existence probability per NB" may be caused.

In addition, an interesting phenomenon observed from Figure 14 is that, for the carriers 2 and 3 , higher $P_{\mathrm{LoS} / \mathrm{NB}}$ is observed with larger probability than in the case of carrier 1 . This is probably due to the reason that the carriers 2 and 3 might be more widely used in the pico-cellular NBs than the 


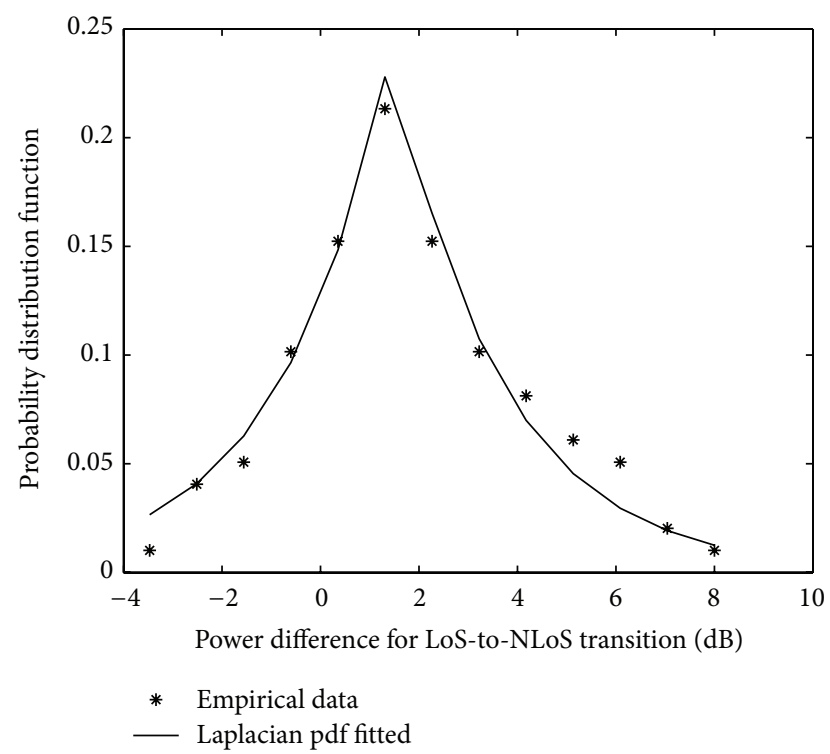

Figure 15: The empirical pdf and fitted Laplacian pdf for the power difference of the channels before and after the LoS-to-NLoS transition.

carrier 1 . Since more LoS users exist in the pico-cellular NBs than other types of NBs [45], the carriers 2 and 3 exhibit higher probability than the carrier 1 for larger $P_{\mathrm{LoS} / \mathrm{NB}}$.

4.5. Channel Characterization of LoS-NLoS Transition. Power diversity techniques used in the NOMA systems mitigate the multipath fading by adapting the transmission power, for example, allocating low power for UE in LoS scenarios and higher power for UE in NLoS scenarios. Designing such techniques requires accurate modeling of the power difference between the LoS and NLoS channels, particularly during the transition process. To design the transmission techniques used in the LoS and NLoS scenarios specifically, adaptation operations, such as switching the techniques or modifying operational parameters, are necessary when the channel changes from the LoS scenario to the NLoS scenario or vice versa. Thus, it is necessary to characterize the channel variations during the period of LoS-to-NLoS or NLoS-to-LoS transition. Measurements were conducted around multiple crosses along Nanjing Road to acquire the downlink data for 1 minute while the receiver was moving. The transitions between LoS and NLoS channels are identified and the interested parameters are analyzed.

4.5.1. Power Difference at LoS-NLoS Transition. Figure 15 demonstrates that the empirical pdf of the power differences $\Delta P_{\mathrm{LoS}-\mathrm{NLoS}}=P_{\mathrm{LoS}}-P_{\mathrm{NLoS}}$ at LoS-to-NLoS transition points. The fitted analytical graph is also illustrated. It can be observed from Figure 15 that the majority of the distribution of $\Delta P_{\text {LoS-NLoS }}$ is located above $0 \mathrm{~dB}$ in the abscissa. The Laplace distribution with its parameters $\mu=1.42 \mathrm{~dB}$ and $b=$ 2.22 fits well with the empirical data. Furthermore, the observation that $\Delta P_{\text {LoS-NLoS }}$ is less than $0 \mathrm{~dB}$ indicates that, for some transitions, the NLoS scenarios have higher received
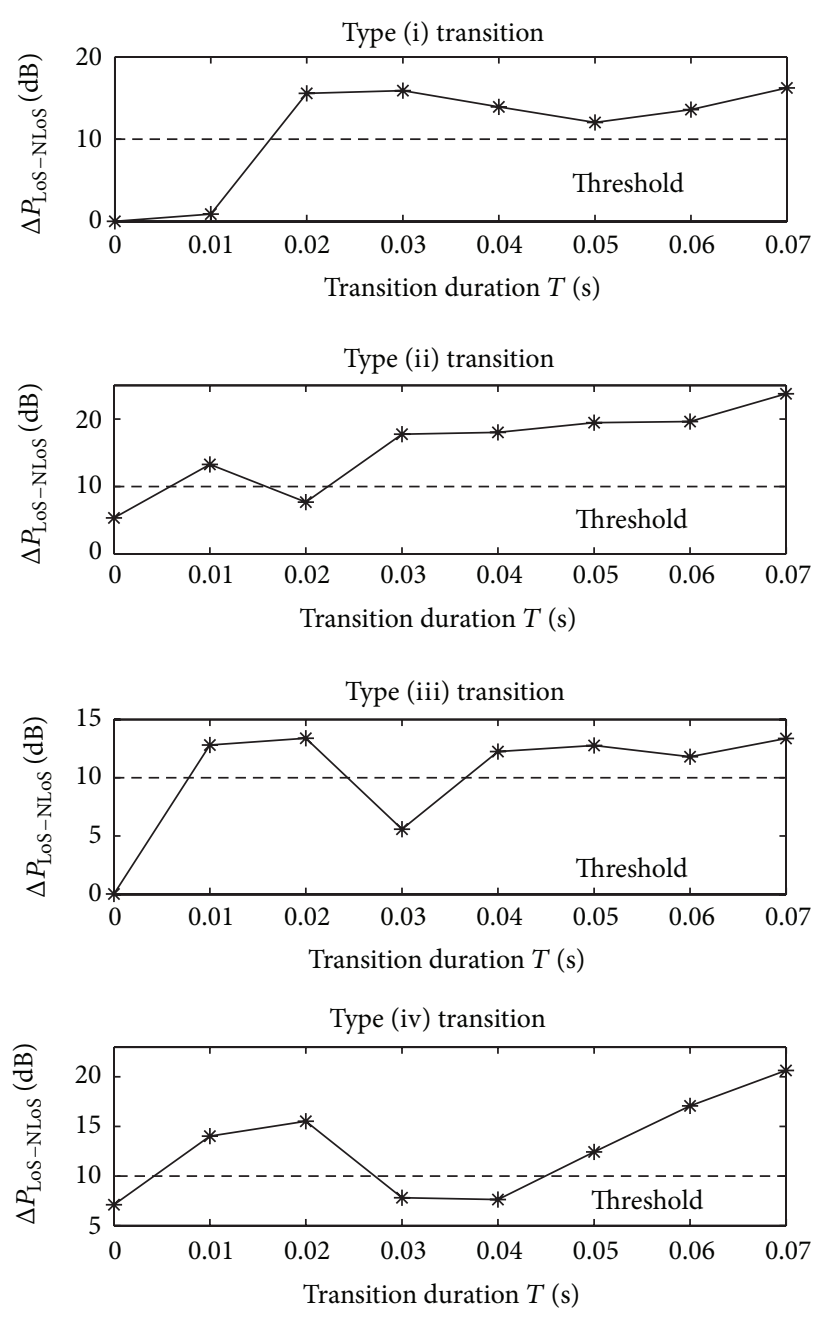

FIgURE 16: Examples of the four types of transition behavior.

power than the LoS scenarios. This phenomenon may be due to the fact that, for some locations such as the corners of streets, when a UE turns its moving direction significantly, the LoS-to-NLoS transition occurs, and the constellation of multipath propagation may include new NLoS paths which add more power to the channel than the power lost due to the absence of the LoS path. This is different with the obstruction cases where the overall propagation constellation is retained with only the LoS path absent due to blockage.

4.5.2. LoS-to-NLoS Transition Duration. The LoS-to-NLoS transition duration $T$ is defined as the time spent on the period from the starting of transition to the moment that the NLoS channel begins to stabilize. Here the channel is considered stabilized if the LoS or NLoS status is maintained for no less than 10 frames. The observations show that all LoS-to-NLoS transitions can be categorized into four types depending on the times of back-and-forth between LoS and NLoS scenarios. Figure 16 illustrates examples for the four transition types. Type (i) is referred to as the case where a LoS channel changes to a NLoS channel which stabilizes immediately. For such cases, $T$ is regarded to be 1 frame. Type (ii) 


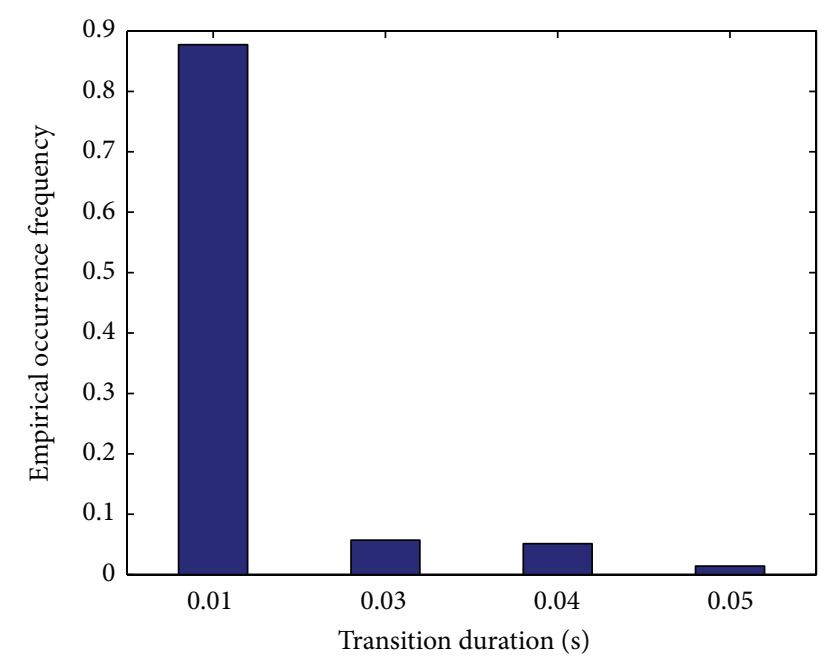

FIGURE 17: The empirical occurrence frequency of transition duration from LoS points to NLoS points or NLoS points to LoS points.

denotes the situation that, after the transition starts, the NLoS channel changes back to the LoS scenario once and then transits again to the NLoS scenario. For this case, $T$ is considered to be 3 frames. Type (iii) is similar with type (ii) but the difference is that, during the transition, the NLoS scenario lasts for two frames before transiting back to the LoS. Type (iv) is similar with type (iii) except for the fact that the LoS channel remains for two frames before transiting to the NLoS scenario in the second time. For the latter two types, $T$ is considered to be 4 and 5 frames, respectively. Figure 17 depicts the empirical probability of $T$ for the four types considered. It can be observed that the type I transition occurs with the highest probability of 0.9 among all types. Only a small amount of transitions undergoes the fluctuations in types (ii) to (iv) which cost a longer time for channel being stabilized. These results show that, for the walking speed in a city canyon environment, most of the LoS-to-NLoS transitions are accomplished rapidly in one frame, that is, $10 \mathrm{~ms}$. Longer transitions lasting for more than one frame can be neglected when designing systems for their low occurrence probabilities.

\section{Conclusions}

A channel measurement campaign has been conducted aiming at characterizing LoS/NLoS channels by using the downlink signals in a commercial UMTS network for a pedestrian zone in Nanjing Road, Shanghai. Techniques were developed for extracting the channel impulse responses, and multipath parameters were estimated by using the SAGE algorithm. Channels observed were categorized into LoS and NLoS by using a novel multipath-based LoS detection method. A map overlaid by the channel properties observed at multiple locations demonstrated that LoS channels mostly exist in open areas, while NLoS channels are dominant in the city canyons with densely distributed skyscrapers. Statistical models for the fading coefficients were first established, which have shown that the existence of the LoS path can bring $6 \mathrm{~dB}$ gain on average and reduce the gain variation by $5 \mathrm{~dB}$. Additionlly, the statistics of some newly defined parameters were also investigated, which include the distributions of the LoS and NLoS life-distance, the LoS existence probability per user equipment and per NB, power difference at the LoSto-NLoS transition, and the transition duration. Important findings were discovered; for example, the LoS and NLoS life-distances are usually less than $10 \mathrm{~m}$, the LoS-to-NLoS transition can be accomplished less than $10 \mathrm{~ms}$, and so forth. These characteristics are important for the design and preference evaluation of the techniques or systems adaptive to LoS and NLoS scenarios.

\section{Conflict of Interests}

The authors declare that there is no conflict of interests regarding the publication of this paper.

\section{Acknowledgments}

The authors wish to acknowledge Dr. Junhe Zhou, Tongji University, for his valuable comments on the paper. This work is jointly supported by Huawei Technology Company in the research Project "Analysis and Verification of Channel Fingerprinting Characteristics in In-Service UMTS Networks" (YBWL2010KJ010), Huawei innovation research Project "High Dimensional MIMO Channel Estimation, Modeling, and Measurement Combined with Antenna Status," the Science and Technology Commission of Shanghai Municipality "System Design and Demo-Construction for Cooperative Networks of High-Efficiency 4G Wireless Communications in Urban Hot-Spot Environments" (13510711000), and the Key Program of National Natural Science Foundation of China (Grant no. 61331009).

\section{References}

[1] Universal Mobile Telecommunications System (UMTS); Spacial Channel Model for Multiple Input Multiple Output (MIMO) Simulations (3GPP TR 25.996 Version 8.0.0 Release 8), ETSI Std.

[2] WINNER II interim channel models, IST-4-027756 WINNER D1.1.1 Std.

[3] D. Gesbert, M. Shafi, D. Shiu, P. J. Smith, and A. Naguib, "From theory to practice: an overview of MIMO space-time coded wireless systems," IEEE Journal on Selected Areas in Communications, vol. 21, no. 3, pp. 281-302, 2003.

[4] M. Coldrey, H. Koorapaty, J. Berg et al., "Small-cell wireless backhauling: a non-line-of-sight approach for point-to-point microwave links," in Proceedings of the 76th IEEE Vehicular Technology Conference (VTC Fall '12), September 2012.

[5] S. Mukherjee, WiMAX Antennas Primer-A Guide to MIMO and Beamforming, 2010.

[6] B. Furht and S. A. Ahson, Long Term Evolution: 3GPP LTE Radio and Cellular Technology, 2009.

[7] Y. Saito, Y. Kishiyama, A. Benjebbour, T. Nakamura, A. Li, and K. Higuchi, "Non-orthogonal multiple access (NOMA) for future radio access," in Proceedings of the IEEE 77th Vehicular Technology Conference (VTC '13), 2013. 
[8] K. Higuchi and Y. Kishiyama, "Non-orthogonal access with random beamforming and intra-beam SIC for cellular mimo downlink," in Proceedings of the IEEE 78th Vehicular Technology Conference (VTC '13), pp. 1-5, Las Vegas, Nev, USA, September 2013.

[9] J. Umehara, Y. Kishiyama, and K. Higuchi, "Enhancing user fairness in non-orthogonal access with successive interference cancellation for cellular downlink," in Proceedings of the IEEE 14th International Conference on Communication Systems (ICCS '12), pp. 324-328, Singapore, November 2012.

[10] D. Jiang and L. Delgrossi, "IEEE 802.11p: towards an international standard for wireless access in vehicular environments," in Proceedings of the 67th IEEE Vehicular Technology Conference (VTC '08), pp. 2036-2040, May 2008.

[11] X. Cheng, C. Wang, B. Ai, and H. Aggoune, "Envelope level crossing rate and average fade duration of nonisotropic vehicleto-vehicle Ricean fading channels," IEEE Transactions on Intelligent Transportation Systems, vol. 15, no. 1, pp. 62-72, 2014.

[12] X. Cheng, Q. Yao, M. Wen, C. Wang, L. Song, and B. Jiao, "Wideband channel modeling and intercarrier interference cancellation for vehicle-to-vehicle communication systems," IEEE Journal on Selected Areas in Communications, vol. 31, no. 9, pp. 434-448, 2013.

[13] V. Nurmela, T. Jämsä, P. Kyösti, V. Hovinen, and J. Medbo, "Channel modelling for device-to-device scenarios," IC 1004 $\mathrm{TD}(13) 08009,2013$.

[14] D. W. Matolak, "Channel modeling for vehicle-to-vehicle communications," IEEE Communications Magazine, vol. 46, no. 5, pp. 76-83, 2008.

[15] Y. Zhang, R. Yu, M. Nekovee, Y. Liu, S. Xie, and S. Gjessing, "Cognitive machine-to-machine communications: visions and potentials for the smart grid," IEEE Network, vol. 26, no. 3, pp. 6-13, 2012.

[16] J. Borras, P. Hatrack, and N. B. Mandayam, "Decision theoretic framework for NLOS identification," in Proceedings of the 48th IEEE Vehicular Technology Conference (VTC '98), pp. 1583-1587, May 1998.

[17] M. Wylie-Green and J. Holtzman, "Locating mobile stations with non-line-of-sight measurements," Advances in Wireless Communications, p. 359, 1998.

[18] S. Venkatraman, J. Caffery Jr., and H.-. You, "Location using LOS range estimation in NLOS environments," in Proceedings of the 55th IEEE Vehicular Technology Conference (VTC '02), vol. 2, pp. 856-860, May 2002.

[19] J. Schroeder, S. Galler, K. Kyamakya, and K. Jobmann, "NLOS detection algorithms for ultra-wideband localization," in Proceedings of the 4th Workshop on Positioning, Navigation and Communication (WPNC '07), pp. 159-166, March 2007.

[20] A. Maali, H. Mimoun, G. Baudoin, and A. Ouldali, "A new low complexity NLOS identification approach based on UWB energy detection," in Proceedings of the IEEE Radio and Wireless Symposium (RWS '09), pp. 675-678, San Diego, Calif, USA, January 2008.

[21] A. Lakhzouri, E. S. Lohan, R. Hamila, and M. Rentors, "Extended Kalman filter channel estimation for line-of-sight detection in WCDMA mobile positioning," EURASIP Journal on Applied Signal Processing, vol. 2003, no. 13, pp. 1268-1278, 2003.

[22] F. Benedetto, G. Giunta, A. Toscano, and L. Vegni, "Dynamic LOS/NLOS statistical discrimination of wireless mobile channels," in Proceedings of the 65th IEEE Vehicular Technology Conference (VTC'07), pp. 3071-3075, 2007.
[23] T. Svantesson and J. Wallace, "Statistical characterization of the indoor mimo channel based on los/nlos measurements," in Proceedings of the 36th Asilomar Conference on Signals, Systems and Computers, vol. 2, pp. 1354-1358, IEEE, 2002.

[24] K. Yu, M. Bengtsson, B. Ottersten, D. McNamara, P. Karlsson, and M. Beach, "Modeling of wide-band MIMO radio channels based on NLoS indoor measurements," IEEE Transactions on Vehicular Technology, vol. 53, no. 3, pp. 655-665, 2004.

[25] C. Bergljung and P. Karlsson, "Propagation characteristics for indoor broadband radio access networks in the $5 \mathrm{GHz}$ band," in Proceedings of the 9th IEEE International Symposium on Personal, Indoor and Mobile Radio Communications (PIMRC '98), vol. 2, pp. 612-616, September 1998.

[26] K. Yu, M. Bengtsson, B. Ottersten, D. McNamara, P. Karlsson, and M. Beach, "Second order statistics of NLOS indoor MIMO channels based on $5.2 \mathrm{GHz}$ measurements," in Proceedings of the IEEE Global Telecommunicatins Conference (GLOBECOM '01), pp. 156-160, November 2001.

[27] H. Yang, P. F. M. Smulders, and M. H. A. J. Herben, "Frequency selectivity of $60 \mathrm{GHz}$ LOS and NLOS indoor radio channels," in Proceedings of the 63rd IEEE Vehicular Technology Conference (VTC '06), vol. 6, pp. 2727-2731, July 2006.

[28] Z. Lin, X. Peng, K. B. Png, and F. Chin, "Kronecker modelling for correlated shadowing in UWB MIMO channels," in Proceedings of the IEEE Wireless Communications and Networking Conference (WCNC '07), pp. 1585-1589, March 2007.

[29] J. C. Liberti and T. S. Rappaport, "Statistics of shadowing in indoor radio channels at 900 and $1900 \mathrm{MHz}$," in Proceedings of the IEEE Military Communications Conference (MILCOM '92), pp. 1066-1070, 1992.

[30] J. E. Berg, R. Bownds, and F. Lotse, "Path loss and fading models for microcells at $900 \mathrm{MHz}$," in Proceedings of the 42th IEEE Vehicular Technology Conference (VTC '92), pp. 666-671, 1992.

[31] S. Y. Tan and H. S. Tan, "Propagation model for microcellular communications applied to path loss measurements in Ottawa City streets," IEEE Transactions on Vehicular Technology, vol. 44, no. 2, pp. 313-317, 1995.

[32] V. Erceg, L. J. Greenstein, S. Y. Tjandra et al., "Empirically based path loss model for wireless channels in suburban environments," IEEE Journal on Selected Areas in Communications, vol. 17, no. 7, pp. 1205-1211, 1999.

[33] E. Green and M. Hata, "Microcellular propagation measurements in an urban environment," in Proceedings of the IEEE Personal, Indoor and Mobile Radio Communications (PIMRC '91), pp. 324-328, 1991.

[34] Y. Oda, K. Tsunekawa, and M. Hata, "Advanced LOS path-loss model in microcellular mobile communications," IEEE Transactions on Vehicular Technology, vol. 49, no. 6, pp. 2121-2125, 2000.

[35] G. Bauer and R. Jakoby, "Analysis and estimation of line-of-sight coverage in urban areas for fixed broadband wireless access systems," in Proceedings of the 10th European Conference on Wireless Technology (ECWT '07), pp. 229-232, October 2007.

[36] J. Yu, X. Yin, J. Chen et al., "Channel maps and stochastic models in elevation based on measurements in operating networks," in Proceedings of the IEEE Wireless Communications and Signal Processing (WCSP '13), pp. 1-6, 2013.

[37] M. Ylitervo and M. Vayrynen, "Methods for making active channel measurements in a personal base station environment," US Patent 5726 981, 1998.

[38] K. Pentikousis, M. Palola, M. Jurvansuu, and P. Perälä, "Active goodput measurements from a public 3G/UMTS network," IEEE Communications Letters, vol. 9, no. 9, pp. 802-804, 2005. 
[39] J. Potman, F. W. Hoeksema, and C. H. Slump, "Adjacent channel interference in UMTS networks, prorisc," in Proceedings of the 17th Annual Workshop on Circuits, Systems and Signal Processing, Veldhoven, The Netherlands, 2006.

[40] X. Yin, N. Zhang, Z. Zhong et al., "System-level channel modeling based on active measurements from a public 3G/UMTS network," in Proceedings of the 78th IEEE Vehicular Technology Conference (VTC '13), pp. 1-5, 2013.

[41] “USRP n210 datasheet," Tech. Rep., https://www.ettus.com/product/details/UN210-KIT.

[42] Technical Specification Group Radio Access Network, Physical Channels and Mapping of Transport Channels onto Physical Channels (FDD)(Release 9), 3rd Generation Partnership Project 3GPP TS 25.211 V9.2.0 (2010-09) Std.

[43] B. H. Fleury, M. Tschudin, R. Heddergott, D. Dahlhaus, and K. I. Pedersen, "Channel parameter estimation in mobile radio environments using the SAGE algorithm," IEEE Journal on Selected Areas in Communications, vol. 17, no. 3, pp. 434-450, 1999.

[44] A. Goldsmith, Wireless Communications, Cambridge University Press, 2005.

[45] L. Thiele, M. Peter, and V. Jungnickel, "Statistics of the Ricean k-factor at $5.2 \mathrm{GHz}$ in an urban macro-cell scenario," in Proceedings of the 17th IEEE International Symposium on Personal, Indoor and Mobile Radio Communications (PIMRC '06), pp. 1-5, September 2006. 

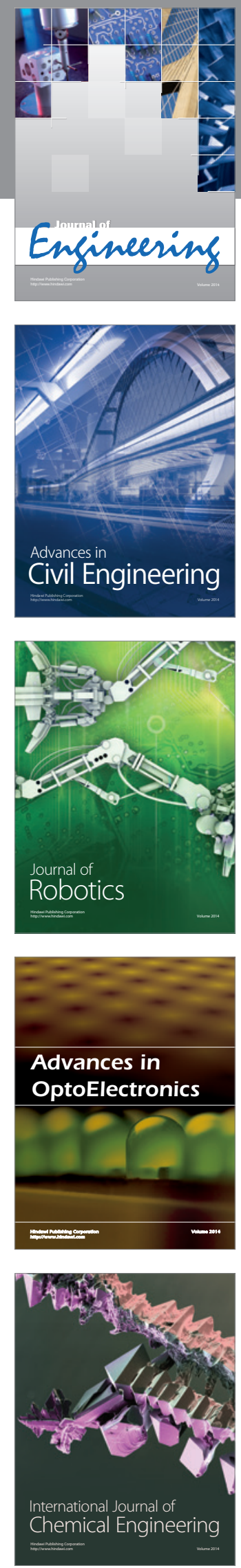

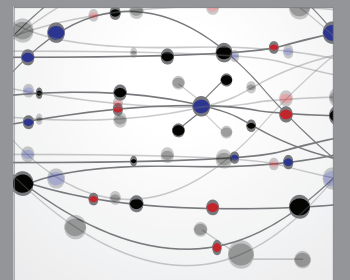

The Scientific World Journal
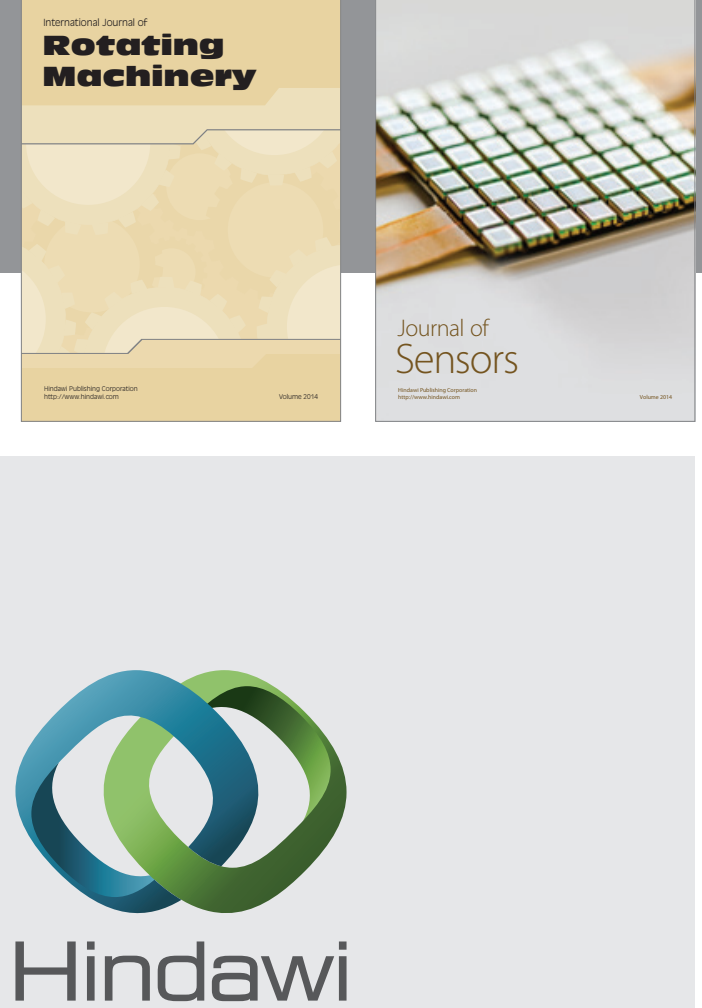

Submit your manuscripts at http://www.hindawi.com
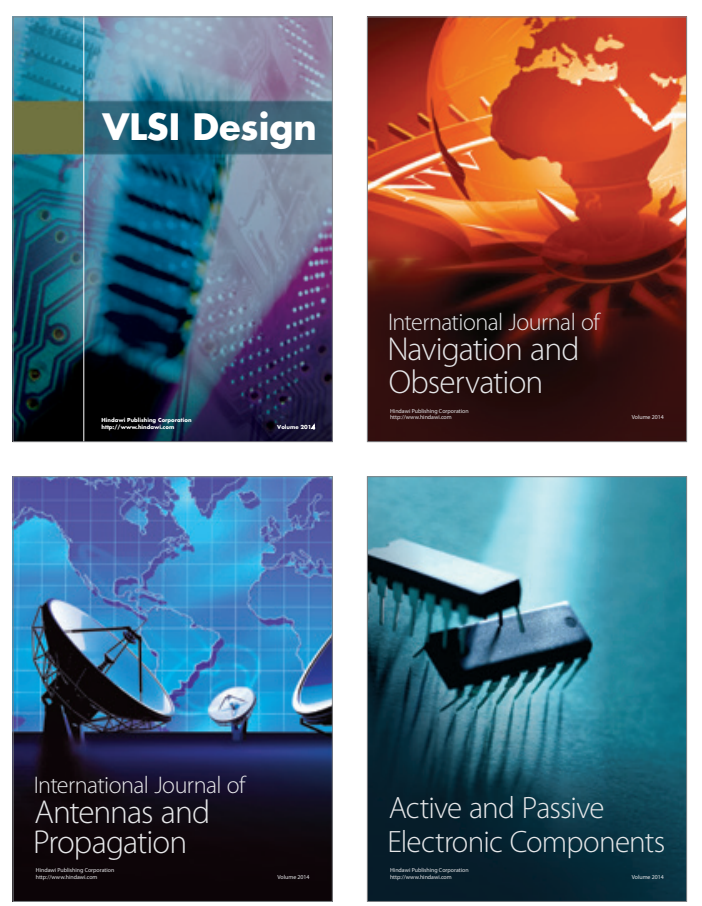
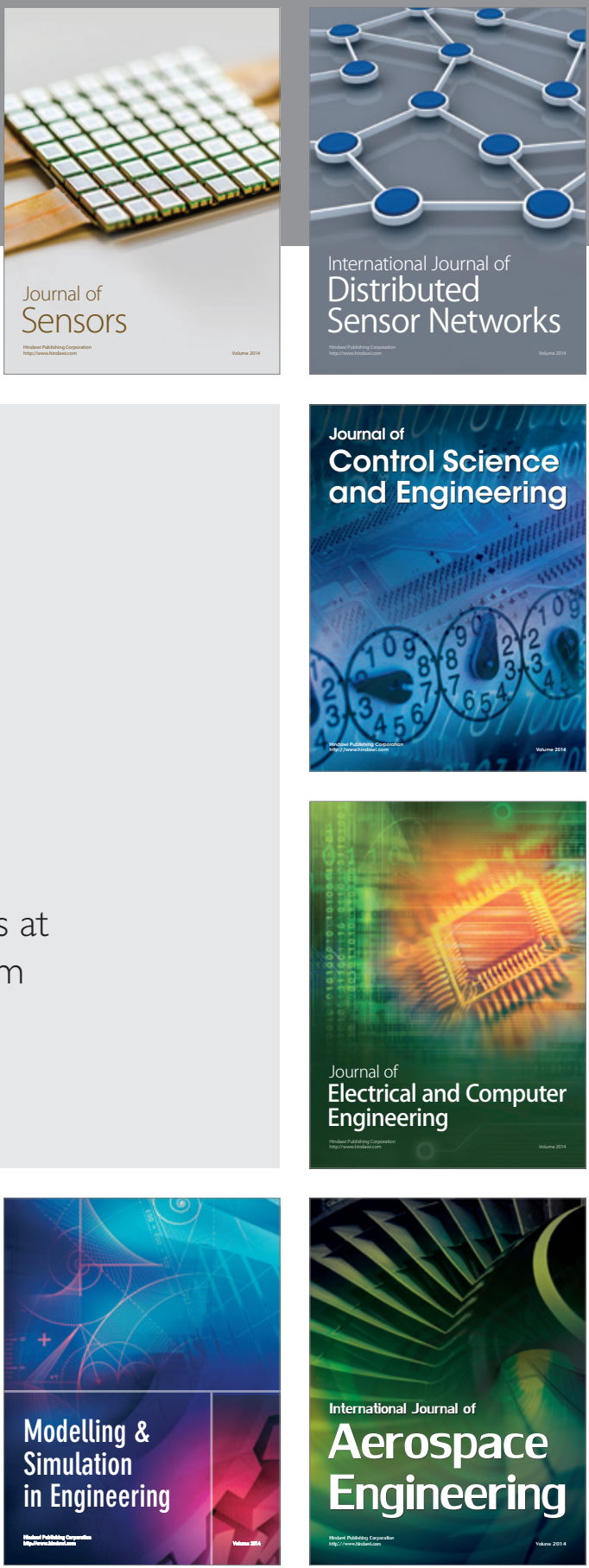

Journal of

Control Science

and Engineering
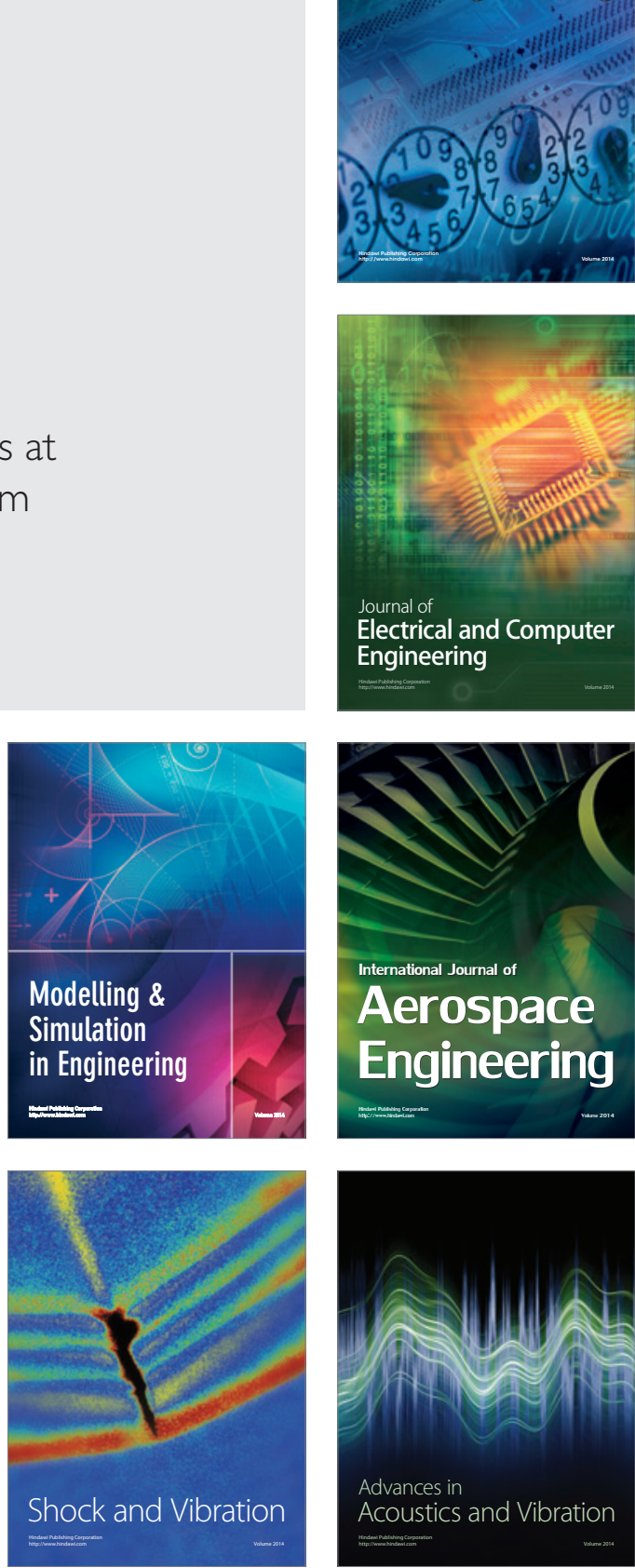